CYSTIC FIBROSIS

\title{
Recovery of Burkholderia cenocepacia strain PHDC from cystic fibrosis patients in Europe
}

\author{
T Coenye, T Spilker, A Van Schoor, J J LiPuma, P Vandamme
}

Thorax 2004;59:952-954. doi: 10.1136/thx.2003.019810

\begin{abstract}
See end of article for authors' affiliations

....................

Correspondence to: Dr T Coenye, Laboratory of Microbiology, Ghent University, K L Ledeganckstraat 35 B-9000 Ghent, Belgium; Tom.Coenye@ugent.be
\end{abstract}

Received 4December 2003 Accepted 26 June 2004

\begin{abstract}
Background: Burkholderia cenocepacia can cause life threatening respiratory tract infections in patients with cystic fibrosis (CF) and has a significant impact on survival. There is extensive evidence for patient to patient spread and nosocomial transmission of this organism, and several widespread $B$ cenocepacia strains have been described including the transatlantic ET12 clone. A study was performed to compare $B$ cenocepacia isolates recovered from CF patients receiving care in several European countries and strains isolated from other clinical samples and the environment, with reference isolates from the epidemic $B$ cenocepacia strain PHDC which has so far only been recovered from CF patients and soil in the USA. Methods: A large collection of $B$ cenocepacia isolates, including a large number recovered from CF patients receiving care in several European countries, Canada and the USA, were genotyped by means of randomly amplified polymorphic DNA typing (RAPD) and rep-PCR using the BOX-A1R primer (BOX-PCR). Results: Nineteen Burkholderia cenocepacia isolates cultured from clinical samples in Europe (18 recently recovered from CF patients in France and Italy and one recovered in 1964 from urine in the UK) showed RAPD fingerprinting patterns that were similar to patterns obtained from isolates of $B$ cenocepacia strain PHDC. Subsequent analysis of these isolates using BOX-PCR confirmed that the European isolates and strain PHDC represent the same clone.

Conclusion: Strain PHDC represents a second transatlantic B cenocepacia clone capable of colonising patients with CF.
\end{abstract}

\section{METHODS}

The Laboratory of Microbiology at Ghent University (Belgium) has a large collection of $B$ cenocepacia isolates, including a substantial number from CF patients receiving care in several European countries, Canada, and the USA. These were sent by referring clinical microbiology laboratories to confirm identification or were exchanged with other researchers in the framework of collaborative studies on the biodiversity of $B$ cepacia complex isolates. This collection included 14 previously characterised PHDC isolates that were deposited in the US B cepacia Research Laboratory and Repository (Ann Arbor, Michigan). Strains were grown aerobically on tryptic soy agar (Oxoid CM131) and incubated at $37^{\circ} \mathrm{C}$. All isolates were identified in the course of several polyphasic taxonomic studies. ${ }^{10}$ DNA from each isolate was prepared as described previously. ${ }^{11}$ The PCR assay for the detection of insertion sequence IS1363 (specific for $B$ cenocepacia strains ET12 and PHDC) was performed as described previously. " Isolate genotyping by means of randomly amplified polymorphic DNA typing (RAPD), repPCR using the BOX-AlR primer (BOX-PCR) and numerical analysis were performed as described previously. ${ }^{11}$

\section{RESULTS}

We investigated a large number of $B$ cenocepacia isolates recovered from European CF patients and other clinical sources, as well as a large and diverse collection of isolates representing established outbreak strains. Of the total number of 796 isolates investigated, 178 gave a positive result in the IS1363-PCR assay, indicating that they most likely either belong to $B$ cenocepacia strain ET12 or to $B$ cenocepacia strain PHDC. ${ }^{9}$ Molecular typing of these isolates using RAPD fingerprinting identified multiple groups, including a group of 33 isolates containing all 14 PHDC reference isolates and 19 European isolates (data not shown). We have previously shown that RAPD fingerprinting, although a rapid and valuable tool in small scale epidemiological studies, is not always sufficiently reproducible to allow reliable comparisons across a larger number of assays. ${ }^{11}$ We therefore re-examined the group of isolates with DNA profiles resembling PHDC using BOX-PCR fingerprinting, a method more suitable for large scale epidemiological studies. ${ }^{11}$ The European $B$ cenocepacia isolates showed BOX-PCR 


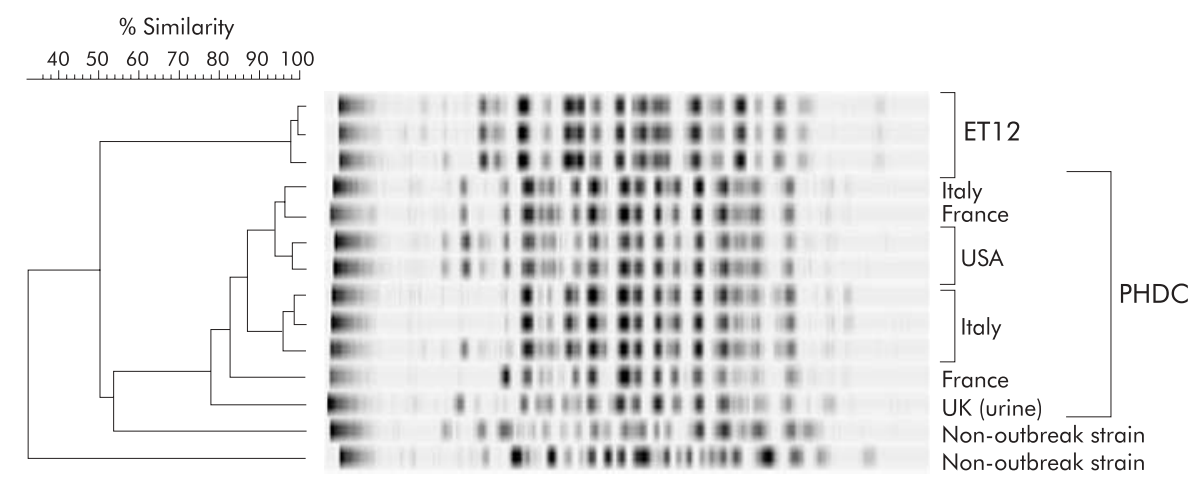

Figure 1 Dendrogram derived from the UPGMA linkage of correlation coefficients between the BOX-PCR fingerprint patterns from B cenocepacia isolates. Epidemic lineage and country of isolation are indicated. All strains were isolated from CF patients unless otherwise indicated.

fingerprint patterns that were very similar to PHDC reference isolates (similarity $>77 \%$, fig 1 ). Of these 19 European PHDC isolates, seven were isolated from CF patients receiving care in France while 11 were recovered from CF patients receiving care in four different Italian CF centres (which were geographically separated). Both groups of isolates were obtained in the course of recent national surveillance studies (P Vandamme, unpublished data). ${ }^{12}$ The remaining isolate was recovered from the urine of a child in the UK in $1964 .{ }^{13}$ All isolates were recovered from different patients.

\section{DISCUSSION}

To date, the best studied $B$ cepacia complex strain is $B$ cenocepacia strain ET12. This strain was found to be predominant among CF patients in Canada and supposedly spread to the UK by patient to patient transmission at CF summer camps. ${ }^{6}{ }^{14} \mathrm{Few}$ other strains infecting large numbers of patients attending more than one or a few centres have been described, but these appear to be geographically more restricted..$^{12}{ }^{15}$ Our data indicate that strain PHDC represents a second transatlantic B cenocepacia clone. To our knowledge, the isolate recovered from urine in the UK in 1964 represents the oldest identified PHDC isolate. The identification of PHDC isolates in clinical samples obtained in different hospitals located in different cities in three different countries in Europe (some cities are located almost $3000 \mathrm{~km}$ apart) over a time span of nearly four decades again confirms that this clone is much more widespread than previously appreciated. ${ }^{9}$ The introduction of infection control measures (including segregation of infected patients) has drastically reduced, but not eliminated, person to person transmission of $B$ cepacia complex organisms, suggesting that new infections may be caused by organisms acquired from the environment. So far, $B$ cenocepacia strain PHDC has been recovered from agricultural soil in the USA; ${ }^{8}$ whether it also can be found in soil in Europe requires further study. It seems unlikely that the epidemiology of PHDC can be explained by patient to patient transmission alone. Its presence in CF patients in Western and Southern Europe and North America suggests acquisition from the environment on a number of occasions, possibly followed by local dissemination by patient to patient contact. A role for inter-patient spread has been shown by previous epidemiological studies. ${ }^{79}$ It should be clear that more work is required to obtain a comprehensive picture of the worldwide epidemiology of $B$ cenocepacia strain PHDC. As detailed epidemiological data were not available for the isolates included in the present study, the epidemiology of this clone needs to be investigated in more detail in future studies.
The present study again highlights the utility of various genotyping methods for $B$ cepacia complex organisms. Although RAPD fingerprinting is easy to perform, its reproducibility can be rather low which may render the interpretation of RAPD pattern differences cumbersome. ${ }^{11}$ BOX-PCR, although somewhat more time consuming, has the advantage of being more reproducible, thus allowing it to be used in global epidemiological investigations involving large numbers of isolates collected over a longer time frame and a wider geographical area. ${ }^{11}$ We have previously shown that results obtained with BOX-PCR are generally in good agreement with those obtained with macrorestriction digests followed by PFGE. ${ }^{11}$ However, PFGE is more time consuming and more expensive than BOX-PCR and, for answering global epidemiological questions (especially those involving larger number of isolates), BOX-PCR might be considered the typing method of choice. ${ }^{11}$ We were able rapidly to screen a large collection of European $B$ cenocepacia isolates and to identify reliably isolates that belonged to the PHDC lineage using a combination of two rapid and inexpensive typing methods.

\section{ACKNOWLEDGEMENTS}

The authors thank E Bingen, S Campana, S Stefani, and G Taccetti for depositing strains in their collection.

\section{Authors' affiliations}

T Coenye, A Van Schoor, P Vandamme, Laboratory of Microbiology, Ghent University, Ghent, Belgium

T Spilker, J J LiPuma, Department of Pediatrics and Communicable Diseases, University of Michigan, Ann Arbor, Michigan, USA

TC and PV are indebted to the Fund for Scientific Research - Flanders (Belgium) for a position as postdoctoral fellow and research grants, respectively. TC also acknowledges the support from the Belgian Federal Government (Federal Office for Scientific, Technical and Cultural Affairs). JJL is supported by a grant from the CF Foundation (US).

\section{REFERENCES}

1 Govan JRW, Deretic V. Microbial pathogenesis in cystic fibrosis: mucoid Pseudomonas aeruginosa and Burkholderia cepacia. Microbiol Rev 1996;60:539-74.

2 LiPuma JJ. Burkholderia cepacia: management issues and new insights. Clin Chest Med 1998; 19:473-86.

3 Coenye T, LiPuma JJ. Molecular epidemiology of Burkholderia species. Fronti Biosci 2003;8:e55-67

4 Coenye T, Vandamme P. Diversity and significance of Burkholderia species occupying diverse ecological niches. Env Microbiol 2003;5:719-29.

5 Vandamme $\mathbf{P}$, Holmes B, Coenye T, et al. Burkholderia cenocepacia sp. nov. - a new twist to an old story. Res Microbiol 2003;154:91-6.

6 Johnson WM, Tyler SD, Rozee KR. Linkage analysis of geographic and clinical clusters in Pseudomonas cepacia infections by multilocus enzyme electrophoresis and ribotyping. J Clin Microbiol 1994;32:924-30.

7 Chen J, Witzmann KA, Spilker T, et al. Endemicity and inter-city spread of Burkholderia cepacia genomovar III in cystic fibrosis. J Pediatr 2001;139:643-9. 
8 LiPuma JJ, Spilker T, Coenye T, et al. An epidemic Burkholderia cepacia complex strain identified in soil. Lancet 2002;359:2002-3.

9 Liu L, Spilker T, Coenye T, et al. Identification by subtractive hybridisation of a novel insertion element specific for two widespread epidemic Burkholderia cepacia genomovar III strains. J Clin Microbiol 2003:41:2471-6.

10 Coenye T, Vandamme P, Govan JRW, et al. Taxonomy and identification of the Burkholderia cepacia complex. J Clin Microbiol 2001;39:3427-36.

11 Coenye T, Spilker T, Martin A, et al. Assessment of genotyping methods for epidemiologic study of Burkholderia cepacia genomovar III. J Clin Microbiol 2002;40:3300-7.
12 Segonds C, Bingen E, Couetdic G, et al. Genotypic analysis of Burkholderia cepacia isolates from 13 French cystic fibrosis centers. J Clin Microbiol 1997;35:2055-60.

13 Stanier RY, Palleroni NJ, Doudoroff M. The aerobic pseudomonads: a taxonomic study. J Gen Microbiol 1966:43:159-271.

14 Govan JRW, Brown PH, Maddison J, et al. Evidence for transmission of Pseudomonas cepacia by social contact in cystic fibrosis patients. Lancet 1993;342:15-9.

15 Coenye T, LiPuma, JJ. Population structure analysis of Burkholderia cepacia genomovar III: varying degrees of genetic recombination characterize major clonal complexes. Microbiology 2003;149:77-88.

\section{LUNG ALERT}

The role of pneumonectomy in non-tuberculous mycobacterial infections

$\Delta$ Shiraishi Y, Nakajima Y, Katsuragi N, et al. Pneumonectomy for nontuberculous mycobacterial infections. Ann Thorac Surg 2004;78:399-403

S urgery has a role in the treatment of patients with atypical mycobacteria who fail medical treatment or have extensive disease localised to one lung. This case series reports over 15 years of experience from the Fukujuji Hospital, Tokyo, reviewing 53 patients who required pulmonary resection for resistant non-tuberculous mycobacterial infections. Of these, 11 underwent pneumonectomy. Patients were selected for pulmonary resection if they met the diagnostic criteria recommended by the American Thoracic Society for non-tuberculous mycobacterial disease and had sufficient cardiopulmonary reserve. The 11 chosen for pneumonectomy had multiple cavities in one lung or destruction of an entire lung. Four of the 11 had evidence of lesions in the contralateral lung; 10 had Mycobacterium avium and one had $M$ abscessus.

There was no operative mortality. A major complication of pneumonectomy is bronchopleural fistula, especially for right pneumonectomy. In this series bronchopleural fistula occurred in three patients $(27 \%)$, all right sided, detected on the chest radiograph before the patient developed symptoms, repaired and without subsequent empyema. These fistulae occurred from 2 weeks to 3 months postoperatively. Other postoperative complications included respiratory failure (1) and empyema (1). All patients became sputum negative after surgery with seven kept on chemotherapy for at least 6 months. The other four did not tolerate chemotherapy. Two patients died, both of respiratory failure, one 11 months postoperatively and the other 4 years later in the presence of recurrent disease.

Pneumonectomy has a role in the treatment of resistant non-tuberculous mycobacterium in patients with one lung severely affected. The majority of patients become sputum negative with continuing chemotherapy and the most common complication is bronchopleural fistula.

G Price Senior House Officer, London Chest Hospital, London, UK; graniaprice@doctors.org.uk 